\title{
HLA class II allele polymorphism in Hungarian patients with primary Sjögren's syndrome
}

\section{A. Kovács, E. Endreffy, I. Petri, L. Kovács \& G. Pokorny}

To cite this article: A. Kovács, E. Endreffy, I. Petri, L. Kovács \& G. Pokorny (2006) HLA class II allele polymorphism in Hungarian patients with primary Sjögren's syndrome, Scandinavian Journal of Rheumatology, 35:1, 75-76, DOI: 10.1080/03009740500287517

To link to this article: https://doi.org/10.1080/03009740500287517

曲 Published online: 12 Jul 2009.

Submit your article to this journal $\pi$

Џ Article views: 24

Q View related articles ¿

4 Citing articles: 6 View citing articles 


\title{
LETTERS
}

\section{HLA class II allele polymorphism in Hungarian patients with primary Sjögren's syndrome}

\author{
A Kovács ${ }^{1}$, E Endreffy ${ }^{2}$, I Petri ${ }^{3}$, L Kovács ${ }^{1}$, G Pokorny ${ }^{1}$ \\ Departments of ${ }^{1}$ Rheumatology, ${ }^{2}$ Paediatrics and ${ }^{3}$ Transfusiology, University of Szeged, Albert Szent-Györgyi Medical and Pharmaceutical \\ Centre and City Hospital, Szeged, Hungary
}

Previous data suggest that human leucocyte antigen (HLA) class II allele polymorphisms are involved in the development of primary Sjögren's syndrome (pSS). HLA DR3, DR2, and DR5 (DRB1*03, *15/16, *11/12) have been found with elevated frequency in pSS and the DR3 positivity correlates with the anti-Ro(SSA) and anti-La(SSB) autoantibody positivities and extraglandular manifestations $(1,2)$. In Greek pSS patients, the incidence of the DR5 allele was significantly increased, whereas no differences in HLA were found between anti-Ro(SSA)-positive and -negative SS patients (3). Finnish pSS patients with the HLA-DRB $1 * 0301-D Q A 1 * 0501-D Q B 1 * 0201$ haplotypes were observed to have significantly higher anti-SSA/Ro and anti-SSB/La antibody levels than did the patients without this haplotype (4). This autoantibody response likewise associated positively with DRB1*03-DQB1*02 and DRB1*03/DRB1*15DQB1*02/DQB1*0602 heterozygosity in a German population (5). In French patients (6), the HLA alleles predisposed to autoantibody secretion without being associated with a clinical outcome (DRB1*15 favoured anti-SSA, and DRB $1 * 03$ both anti-SSA and anti-SSB production). In Norwegian pSS patients, similar HLA allele findings (DRB $1 * 0301$ and DRB $3 * 0101$ alleles) were reported, and these alleles were more closely associated with the presence of anti-Ro autoantibodies than with pSS itself (7).

Our aim was to determine the HLA class II allele polymorphisms, and to analyse the pheno- and genotype correlations in 48 Hungarian pSS patients, who all fulfilled the classification criteria for pSS syndrome (8). Forty-seven females and one male, with a mean age at the time of the examination of 55 years (range 33-83), and 50 healthy blood donors matched for age and sex as controls were studied. HLA-DRB1 was genotyped with the Dynal RELI SSO HLA-DRB kit, DRB1*15/16 was subtyped with the method of Ota et al (9), DQA1 by the method of Ota et al (10), and DQB1 with the INNO-LiPA DQB kit (Norway). A $\chi^{2}$-test with Yates' and Bonferroni's corrections was performed, and odds ratios (ORs) were also calculated.

In the pSS patients, DRB $1 * 03-\mathrm{DQA} 1 * 05011$ DQB $1 * 0201$ proved to be the haplotype of susceptibility, even when Bonferroni's correction was used $(\mathrm{p} \leqslant 0.01$, Table 1). The DRB $1 * 1601$ (OR 3.5) and DQA $1 * 0102$ (OR 2.5) alleles also occurred more frequently $(\mathrm{p} \leqslant 0.05)$, compared with the controls. By contrast, the DRB1*01-DQA1*0101-DQB1*0501, DRB1*04-DQA $1 * 0301-\mathrm{DQB} 1 * 0302$ and DRB1*11/ 12-DQA1*05012-DQB1*0301 haplotypes were less common in the pSS patients $(\mathrm{p}<0.05$ and $\mathrm{p}<0.02$, respectively, Table 1). However, after the use of Bonferroni's correction, the difference did not remain significant.

We analysed the HLA class II alleles in the SS subgroups separated according to the anti-SSA/antiSSB seropositivity and to seronegativity. In the 20 anti-SSB-positive pSS patients, the DRB $1 * 03$ allele frequency was significantly higher than in the 28 antiSSB negative cases $(17 / 40$ vs. $12 / 56, \mathrm{p}<0.05)$, but there was no similar difference between the patients with anti-SSA positivity or anti-SSA negativity (22/66 vs. 6/30, ns). By contrast, the DRB $1 * 15 / 16$ (DR2) alleles were detected significantly more frequently in the anti-SSB-negative than in the antiSSB-positive patients $(18 / 56$ vs. $3 / 40, p<0.05)$, and in the anti-SSA plus anti-SSB-negative patients compared with the anti-SSA plus anti-SSB-positive patients $(8 / 24$ vs. $4 / 34, p=0.02)$.

The DRB $1 * 03$ allele was more common in nine pSS patients who exhibited purpura than in those without this vascular manifestation (9/18 vs. $21 / 78$, ns). By contrast, the DRB $1 * 15 / 16$ alleles were less frequent in the patients with purpura (2/18 vs. $21 / 78$, ns). Eleven pSS patients developed renal tubular acidosis, and DRB1*03 proved to be an allele of susceptibility $(12 / 22$ vs. $18 / 74, \mathrm{p}<0.02)$, and DRB $1 * 11 / 12$ one of resistance $(0 / 22$ vs. $11 / 74, p<0.05)$ as compared with the patients without kidney involvement. Malignant lymphomas developed in five pSS patients (Hodgkin 


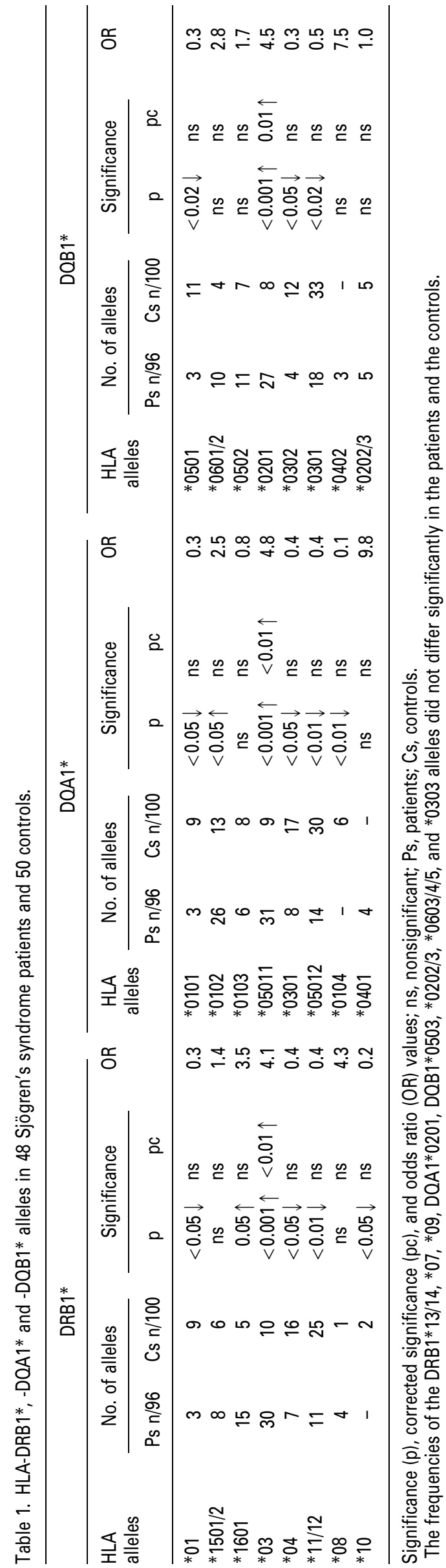

in one and non-Hodgkin in four); all of them carried the DRB1*03 allele (6/10 vs. $24 / 86$ alleles, $\mathrm{p}<0.05$, Yates's correction; ns).

To summarize, in 48 Hungarian pSS patients, DRB1*0301-DQA1*05011-DQB1*0201 proved to be the haplotype of susceptibility. The DRB $1 * 03$ allele exhibited a positive correlation with anti-SSB autoantibody production, the presence of renal tubular acidosis, and the development of malignant lymphoma. The DRB1*15/16 (DR2) alleles were detected more frequently in the anti-SSB-negative patients than in the anti-SSB-positive patients, and in the anti-SSA plus anti-SSB-negative patients than in the anti-SSA plus anti-SSB-positive patients. Our results suggest a model of HLA-restricted presentation of Ro/La peptide determinants in pSS.

\section{References}

1. Rischmuller M, Lester S, Chen Z, Champion G, Van Den Berg R, Beer R. HLA class II phenotype controls diversification of the autoantibody response in primary Sjogren's syndrome. Clin Exp Immunol 1998;111:365-71.

2. Vitali C, Tavoni A, Rizzo G, Neri R, D’Ascanio A, Cristofani $\mathrm{R}$, et al. HLA antigens in Italian patients with primary Sjögren's syndrome. Ann Rheum Dis 1986;45:412-16.

3. Papasteriades CA, Skopouli FN, Drosos AA, Andonopoulos AP, Moutsopoulos HM. HLA-alloantigen associations in Greek patients with Sjögren's syndrome. J Autoimmunity 1988;1:85-90.

4. Kerttula TO, Colin P, Polvi A, Korpela M, Partanen J, Maki M. Distinct immunologic features of Finnish Sjögren's syndrome patients with HLA alleles DRB1*0301, DQA1*501, and DQB1*201. Arthritis Rheum 1996;39:1733-9.

5. Bolstad AI, Wassmuth R, Haga HJ, Jonsson R. HLA markers and clinical characteristics in Caucasians with primary Sjogren's syndrome. J Rheumatol 2001;28:1554-62.

6. Gottenberg JE, Busson M, Loiseau P, Cohen-Solal J, Lepage V, Charron D. In primary Sjogren's syndrome, HLA class II is associated exclusively with autoantibody production and spreading of the autoimmune response. Arthritis Rheum 2003;48:2240-5.

7. Nakken B, Jonsson R, Brokstad KA, Omholt K, Nerland AH, Haga HJ, et al. Associations of MHC class II alleles in Norwegian primary Sjogren's syndrome patients: implications for development of autoantibodies to the Ro52 autoantigen. Scand J Immunol 2001;54:428-33.

8. Vitali C, Bombardieri S, Jonsson R, Moutsopoulos HM, Alexander EL, Carsons SE, et al. Classification criteria for Sjögren syndrome, a revised version of the European criteria proposed by the American-European Consensus Group. Ann Rheum Dis 2002;61:554-58.

9. Ota M, Seki T, Fukushima H, Tsuji K, Inoko H. HLA-DRB1 genotyping by modified PCR-RFLP method combined with group-specific primers. Tissue Antigens 1992;39:187-202.

10. Ota $M$, Seki $T$, Namura $N$, Sugimura $K$, Mizuki $N$, Fukusima H, et al. Modified PCR-RFLP method for HLADPB1 and DQA1 genotyping. Tissue Antigens 1991;38:60-71.

Attila Kovács, Department of Rheumatology, University of Szeged, A. Szent-Györgyi Medical and Pharmaceutical Centre and City Hospital, H-6724 Szeged, Kossuth L. sgt. 42, Hungary. E-mail: kovacsa@szegedkorhaz.hu

Received 10 May 2005

Accepted 31 July 2005 